\title{
Civilisations
}

Revue internationale d'anthropologie et de sciences

humaines

$41 \mid 1993$

Mélanges Pierre Salmon II

\section{Les Turkana : aperçu général d'une société pastorale}

\section{Patrick Wymeersch}

\section{OpenEdition \\ Journals}

Édition électronique

URL : http://journals.openedition.org/civilisations/1691

DOI : 10.4000/civilisations. 1691

ISSN : 2032-0442

\section{Éditeur}

Institut de sociologie de l'Université Libre de Bruxelles

\section{Édition imprimée}

Date de publication : 1 septembre 1993

Pagination : 83-102

ISBN : 2-87263-094-5

ISSN : 0009-8140

Référence électronique

Patrick Wymeersch, «Les Turkana : aperçu général d'une société pastorale », Civilisations [En ligne],

41 | 1993, mis en ligne le 28 juillet 2009, consulté le 30 avril 2019. URL : http://

journals.openedition.org/civilisations/1691; DOI : 10.4000/civilisations.1691

Ce document a été généré automatiquement le 30 avril 2019.

(c) Tous droits réservés 


\title{
Les Turkana : aperçu général d'une société pastorale
}

\author{
Patrick Wymeersch
}

\section{Introduction}

1 En dehors des Maasai, dont la réputation passe les frontières, le Kenya est une mosaïque d'ethnies, fières jusque dans leur dénuement. Au Kenya, pays grand comme la France, à peine $10 \%$ des terres sont cultivables. La compétition entre le mode de vie agricole et sédentaire et le mode de vie pastoral et nomade y ranime parfois les vieux conflits ethniques. L'équilibre entre les agriculteurs de langue bantoue et les nomades nilotes et couchites reste précaire.

2 Les Turkana, classifiés sous le vocable Nilotes des Plaines, sont aux environs de 250000 et habitent le nord-ouest du pays dans une région extrêmement aride et désertique entre le lac qui porte le nom de leur ethnie et l'Ouganda. C'est une région qui appartient à la zone du climat sahélien : désert, caillasse, monticules de roches noires et, çà et là, quelques épineux, des doums, de la brousaille, des arbustes chétifs et torturés par le vent. Les points d'eau sont rares, espacés, parfois taris.

3 Demeurés longtemps en dehors de toute intrusion de l'état moderne - d'abord sous la colonie et ensuite sous le Kenya indépendant - les Turkana, pasteurs nomades parfaitement adaptés à un environnement hostile, ont pourtant dû, à la suite de la grave sécheresse de 1961, diversifier leur économie pastorale. Certains sont devenus pêcheurs.

4 Le lac Turkana est un phénomène extraordinaire de la nature. Situé au milieu d'un des déserts les plus arides et les moins peuplés d'Afrique de l'Est, il s'étend sur près de 400 kilomètres de longueur et de 60 de largeur. Il est alimenté par des chutes de pluies qui n'excèdent pas quelques centimètres par an et par l'Omo, venant d'Ethiopie.

5 Les Turkana du lac dépendent du palmier doum aux feuilles pointues qui sortent directement du tronc comme d'énormes doigts. C'est le seul arbre qui réussit à vivre dans 
cette région. On emploie le doum à tous les usages possibles. Son bois léger et spongieux sert à la fabrication des radeaux. La tige des feuilles est employée pour faire les nasses à poisson et de fines lanières d'écorce servent de ficelle. Les feuilles constituent le chaume du toit des huttes et l'on mange les fruits.

La pêche a connu un certain développement aux environs de Kalakol, avec la création de coopératives. Cependant, le but de ce peuple est de renouer, dès la constitution d'un nouveau cheptel, avec le mode de vie pastoral.

7 Tous les récits traditionnels concordent pour dire que les Turkana sont originaires $d u$ Nord-Est de l'Ouganda, dans la région de Dodos. La date de leur dispersion au Kenya est certainement antérieure au $17^{\text {ème }}$ siècle. Ils repoussèrent les Maasai et d'autres ethnies vers le sud et le sud-est.

8 A l'instar des Couchites et à l'inverse des Maasai, les Turkana ne pratiquent pas la circoncision et la clitoridectomie. Nomades perpétuellement en quête de pâturages aux confins du désert, les Turkana sont aussi de redoutables guerriers et voleurs de vaches. Pour ces nomades, le bétail, les points d'eau et les maigres pâturages qui font cruellement défaut pendant la sécheresse, sont l'enjeu journalier.

\section{Les structures sociales}

9 La filiation est patrilinéaire (awi), mais la maisonnée maternelle (ekal) est importante pour un fils désireux de se marier et constituer les biens matrimoniaux qu'il devra remettre à la parenté de sa future épouse.

10 Le jour de son mariage, l'homme donne en cadeau à sa femme une dizaine de têtes de bétail. Ces bêtes lui appartiendront désormais. Mais comme bien souvent le montant des bêtes reçues de la mère biologique est insuffisant, les guerriers constituent des bandes de la même classe d'âge en vue de razzier les ethnies voisines (surtout Pokot et Toposa) afin de se procurer le bétail nécessaire pour leur mariage.

11 L'unité sociale minimale est le kraal (awi) [à remarquer que les termes de parentés sont souvent des termes architecturaux] constitué par un homme et ses frères, tous mariés avec une ou plusieurs épouses ainsi que leurs enfants qui, à leur tour, peuvent être mariés et avoir aussi déjà des enfants. Les affaires privées (administration) sont traitées par l'âné du kraal.

L'unité économique minimale est également le kraal, car nous avons à faire à une économie pastorale autarcique. Cependant, le chef du kraal entretient des relations d'amitiés avec un homme d'un kraal voisin: on se donne régulièrement et mutuellement des têtes de bétail en usufruit. Le système à un double but :

- nouer et/ou entretenir des relations de bon voisinage dans une région très hostile où survivre est constamment à l'ordre du jour. On peut ainsi faire appel à l'ami en cas de disette ;

- à cause du 'sport favori', le vol de bétail, chaque individu essaie de se préserver du pire en installant une partie de son troupeau chez un ami, dans l'espoir que le vol n'aura pas lieu chez lui. De cette façon l'homme peut plus facilement se reconstituer un troupeau, signe infaillible de richesse et de statut social.

13 Les Turkana sont acéphales. Du point de vue politique (les affaires publiques du groupe), l'unité maximale est l'adakar, c'est-à-dire littéralement 'la région géographique où les 
animaux des différents kraals broutent en commun'. L'adakar varie d'année en année, mais est toujours habité par les mêmes gens des différents awi. Le camp de base, awi napolon, est situé dans l'adakar et est habité de façon plus ou moins continue par les plus vieux et les plus jeunes enfants. Les jeunes guerriers nomadisent parfois très loin du camp de base. Ils habitent alors un awi abor ou camp temporaire.

Les membres vivant dans le même adakar ne sont pas nécessairement de la même parenté (famille étendue et clan). Les Turkana choisissent plutôt comme voisins de bons amis que des gens de la parenté. La famille étendue (eowe) est donc divisée territorialement. Il n'y a pas de lignages segmentaires ou fissionnaires. Les clans (ekitela), 28 au total, ne sont pas corporatifs et n'ont plus aucune fonction sociale et politique. Ils ne jouent même plus de rôle dans le mariage dit exogamique.

Chaque adakar possède des puits d'eau creusés dans le lit asséché d'une rivière. L'adakar peut refuser l'eau à des gens de passage si une demande explicite n'est pas formulée.

Le rituel politico-religieux le plus important est l'akipeyore. C'est une communion de tous les initiés mâles de l'adakar qui se réunisent toutes les semaines. Chaque homme va, à tour de rôle, offrir un animal en sacrifice. Il est rituellement abattu, grillé et mangé. Cet lors de ce rituel que les vieux traitent des affaires publiques des membres de l'adakar et de ceux des adakar voisins.

\section{L'art corporel}

17 La première richesse de cette société c'est, évidemment, le bétail qui assure sa subsistance. Tout se structure, s'organise donc autour et en fonction des animaux. Les guerriers passent une partie de leur vie à pousser les troupeaux de pâturages en points d'eau. Ils mènent, à travers des déserts interminables, une existence austère, presque ascétique. Ils sont armés comme leurs ancêtres : lance et massue ne les quittent jamais. La garde du petit bétail, chèvres et moutons, est confiée aux jeunes enfants. Le pacage les entraîne moins loin du camp de base.

Les Turkana se divisent en petits groupes familiaux extrêmement mobiles pour tirer leur maigre subsistance d'une terre hostile à la végétation éparse. Ils parcourent ainsi des distances variées et ne possèdent qu'un minimum de biens matériels qu'ils doivent être capables de rassembler aisément dès que nécessaire, afin de migrer sans encombrement. Tout ce qu'ils possèdent doit être porté à dos d'âne.

19 Leurs habitations sont de simples structures de branchages et de peaux d'animaux. Aux alentours du lac, les huttes ressemblent à des ruches faites de palmiers doums enfoncés dans le sable. Chaque homme a plusieurs femmes, et chaque femme habite une hutte avec ses enfants biologiques. Dans ce contexte, l'architecture et autres expressions artistiques qui exigent un mode de vie plus stable, tels que le moulage des métaux ou la sculpture sur bois, n'ont guère l'occasion de se développer ; c'est donc sur eux-mêmes que les gens ont dirigé leur créativité, leur besoin d'embellir et de décorer.

20 Les Turkana accordent une importance particulière à la forme naturelle du corps. Ils portent peu de vêtements, mais rehaussent leurs traits à l'aide de peinture et de graisse, et arborent des coiffures et des couvre-chefs compliqués. Des bijoux soulignent leurs membres élancés, aux gestes gracieux. Cet art sert de langage signalétique. Il renseigne sur l'âge de l'individu, les exploits qu'il a pu accomplir et sa position sociale. 
21 Leurs ornements proviennent en général d'éléments naturels glanés dans leur environnement: os, dents, coquillages, peaux, pierres, argile. A partir de ces simples matériaux, et avec beaucoup d'imagination, ils réussissent à créer de beaux effets dramatiques. Au début du siècle, des perles produites à profusion en Europe se négocient par millions le long de la côte orientale. Elles vont donc vite remplacé certains éléments de décoration naturels, tels que coquilles d'oeuf d'autruche. Les ornements sont très variés et font preuve d'imagination. Des colliers de toutes tailles et de toutes formes, faits de lourdes perles enfilées sont portés par rangs multiples autour du cou. On les recouvre souvent de graisse rouge pour éviter l'échauffement de la peau de la nuque et des épaules et pour les protéger des parasites. On coud également des perles sur les jupes de cuir.

Pour les Turkana, l'art corporel a toujours été un facteur important de l'expression artistique. Les bijoux colorés qui accentuent le mouvement servent à rehausser cet art et permettent des effets artistiques rarement égalés dans les autres parties de l'Afrique. Ces ornements fournissent en outre des renseignements précis sur ceux qui les arborent; savoir les identifier et les comprendre revient par conséquent à mieux connaître les individus.

23 Les femmes Turkana s'habillent de peaux d'animaux, qu'elles passeront plus d'un aprèsmidi à enjoliver sous un soleil brûlant. Les petits enfants ne portent qu'un simple rang de perles, et les adolescentes de minuscules cache-sexes, décorés de perles multicolores ou de coquilles d'oeuf d'autruche. Ces vêtements s'allongent au fur et à mesure qu'elles approchent de l'âge nubile. Elles se cousent des pendentifs de perles et de cauris dans les cheveux.

Les hommes utilisent l'argile pour façonner leurs savantes coiffures. Ils en enduisent leurs cheveux divisés en petites tresses, dont ils forment un chignon à l'arrière du crâne. Ils affirmeront leur élévation sociale en y insérant des plumes d'autruche maintenues par des supports de boyau de vache ou de macramé, que l'on met en place quand l'argile est encore humide. On recompose la structure avec soin tous les trois mois.

Les Turkana se font arracher les dents de la mâchoire inférieure pour obliger celles du haut à saillir d'avantage et pour ne pas être gêné par le labret dans la lèvre inférieure. Dès l'enfance, on perce les oreilles des garçons et des filles en prévision des simples boucles d'oreilles en métal qu'ils porteront fièrement.

Du fait de sa rareté, on n'emploie l'eau que pour la boire, jamais pour se laver. La toilette est faite avec de la graisse dont les Turkana se frottent la peau.

\section{Vivre du troupeau}

Les Turkana sont organisés autour d'un système de classes d'âge, ou générations. Tout individu de sexe masculin traverse obligatoirement une série d'étapes bien définies, qui exigent de lui certains devoirs et un comportement conforme à la tradition. Ces étapes sont au nombre de trois : l'enfance, l'âge de guerrier et l'âge d'aîné. Le passage d'un âge à un autre est toujours marqué par des cérémonies rituelles très élaborées. Pour les femmes, le processus est plus simple: elles passent directement de l'enfance à l'âge adulte au moment de la puberté.

Les migrations peuvent couvrir jusqu'à 200 kilomètres, sur des itinéraires habituels ou occasionnels. Chaque jour, le troupeau se déplace du kraal vers les pâtures, le point d'eau et les terres salées. Tous ces mouvements sont une technique d'élevage, mise au point 
depuis des siècles et fixée par la coutume. Le matin très tôt le bétail part en quête d'herbe, accompagné de ses gardiens ; il parcourt aisément de 10 à 20 kilomètres chaque jour pour revenir le soir au kraal. Quand l'eau se fait rare, quand les troupeaux ont épuisé la végétation environnante, ou simplement quand le campement devient trop sale, les Turkana se remettent en route. Ils installent sur le dos de leurs ânes tous leurs ustensiles de ménage. Dès qu'ils trouvent un nouvel endroit propice à l'établissement d'un autre campement, ce sont les femmes et les enfants qui construisent les habitations. Sur des armatures de branches fléchies, on pose des peaux de bêtes et des feuillages.

Les Turkana élèvent à la fois des vaches, des dromadaires, des chèvres, des moutons et des ânes. Ces animaux leur fournissent le lait, élément crucial de leur alimentation, ainsi que les peaux qui leur permettent de construire leurs habitations, de se vêtir, de s'étendre sur des litières. Ils constituent leur unique source de richesse, leur monnaie d'échange, leur héritage et, par dessus tout, leur religion.

Contrairement aux bovins qui doivent paitre, le dromadaire, comme la chèvre, broute et se nourrit d'épineux et d'écorces rugueuses. Il peut se passer d'eau pendant quinze jours ou plus et, quand il boit, il fait des provisions pour quinze autres jours. Sauf en cas de détresse ou à l'occasion de cérémonies spéciales, les pasteurs Turkana répugnent à abattre leurs dromadaires pour la chair. Quand ils le font, ils la mangent et utilisent la peau pour fabriquer des pots.

31 Ces diverses espèces animales coexistent sur les pâturages et leur garde est confiée à des garçons de divers âges, ce qui est un avantage pour l'éleveur. Toutes ces espèces sont bien adaptées au milieu très difficile. Les Turkana sont très attentifs à la sélection des races, pour maintenir celle qui lui convient.

Dans l'ensemble, le bétail est élevé pour ses multiples produits : le lait, la viande et le sang. La base de la nourriture est le lait, mais il faut laisser leur part aux veaux qu'on engraisse. Les conditions de vie sont si précaires que les hommes ne doivent leur survie qu'au lait et au sang. Pour recueillir ce sang, il faut saillir une veine en plaçant un garrot au cou. Mélangé avec du lait, ce breuvage alimente les habitants du kraal. Le beurre sert d'avantage comme cosmétique que comme nourriture. La bouse séchée sert aux feux de cuisine et de garde; les poils, peaux, cornes, os et urine sont récupérés pour fabriquer toutes sortes d'objets et pour nettoyer les pots.

Le bétail est la seule richesse, un capital qui s'accroît tout seul, en quelque sorte. Sa gestion réclame plus de savoir-faire que de travaux pénibles: l'homme se borne à défendre son troupeau, en le gardant dans un enclos d'épineux durant la nuit. Il creuse des puits et doit puiser pour les abreuvoirs.

La garde d'un troupeau, au milieu d'autres troupeaux et de leurs pasteurs, est affaire de courage et de relations humaines plutôt que de force physique. Il faut se renseigner sur les régions atteintes par la sécheresse ou mieux arrosées, sur le mouvement de nombreux autres troupeaux. Il faut négocier pour éviter les querelles autour des puits et, éventuellement, se défendre contre les razzias ou lancer une expédition pour récupérer son bien ou augmenter son propre troupeau.

De nos jours encore, les Turkana n'abattent pas leurs betes pour vendre la viande aux boucheries, mais seulement à l'occasion des fêtes et rituels religieux. Pour un économiste moderne, c'est un scandale: un capital si important qui reste improductif. En fait, l'économie traditionnelle est orientée non vers le marché, mais vers la satisfaction de besoins humains. Constamment les Turkana ont besoin de leurs animaux comme monnaie 
d'échange dans les transactions sociales en vue d'un mariage et dans les multiples circonstances où la coutume veut qu'on fasse un cadeau.

La merveille d'un troupeau, c'est qu'il s'accroît de lui-même ; quand ce capital augmente, la vie de la famille est assurée, les femmes portent de nombreux colliers en perles multicolores. Malheureusement, un troupeau est fragile, il dépend étroitement de l'environnement. Sans doute résiste-t-il mieux que les plantes cultivées à la sécheresse : le bétail trouve toujours de quoi manger puisqu'il peut se déplacer. Aux aléas du climat s'ajoutent les épizooties : on se souvient encore de la catastrophe que fut la peste bovine des années 1890, qui décima les troupeaux.

Devant ces dangers, le raisonnement du Turkana consiste à laisser croître le troupeau le plus possible et à le disperser autant que la sécurité le permet. Ainsi en cas de catastrophe ou de razzia, tout le troupeau n'est pas perdu, il reste de quoi recommencer un cycle d'expansion. Mais quand les troupeaux se multiplient, ils mettent en danger l'équilibre écologique : les plantes surpâturées n'ont plus le temps de repousser; sur les itinéraires obligés et autour des puits, la couverture végétale est piétinée et détruite. C'est pourquoi les Turkana vont toujours plus loin ; encore faut-il qu'il y ait de l'espace disponible, ce qui pose actuellement des problèmes en cas de sécheresse générale.

\section{Hommes et bêtes en symbiose}

La vie du pasteur n'est pas un métier ordinaire, qu'on exerçerait pour l'argent qu'on en tire; ce n'est pas un gagne- pain pour individus passionnés de vie libre et de grands espaces. C'est par contre une manière d'exister en famille, de génération en génération.

Troupeau et famille se parasitent mutuellement, en ce sens que chacun vit de l'autre. Le troupeau est même conçu comme le double, l'ombre de la famille. Le bétail représente pour les Turkana non seulement la nourriture assurée, mais aussi la possibilité d'acquérir des femmes et donc des enfants.

Plus qu'une affaire économique, la vie pastorale est un système social total. Le troupeau est le fondement de la permanence et de l'accroissement de la famille. C'est un héritage qui vient des ancêtres et qu'il faut transmettre aux descendants. Dans la famille, on connaît tous les noms des vaches, leur généalogie, leurs descendants.

41 La gestion d'un troupeau est une affaire collective, elle suppose qu'on entretient un crédit social. Une famille n'est jamais seule sur son territoire (adakar), elle vit au milieu d'autres éleveurs de la même ethnie, qui ont aussi leurs troupeaux. Cette situation requiert des hommes une grande habilité pour négocier et une position sociale que le chef de famille acquiert et transmet à ses enfants. Il lui faut rassembler des informations sur l'état des pâturages suivant les aléas de la météo, sur la position des autres troupeaux, les espaces libres et les itinéraires praticables. Il lui revient de prendre les décisions pour apaiser les conflits, négocier des alliances et, éventuellement, monter une razzia. Toutes ces choses qui supposent un vaste réseau de communications et de solidarité entre les familles.

La fécondité humaine et animale de laquelle l'avenir dépend, joue un rôle important dans la vie de chaque jour et ses aspects rituels. Par tradition, l'enfant est élevé au sein parfois jusqu'à deux ou trois ans. Au cours des premières années, la mère porte l'enfant sur le dos presque toute la journée. Ils sont, de ce fait, toujours très proches physiquement et il est rare d'entendre un bébé pleurer par besoin de tendresse, de contact maternel. 

le Turkana, la véritable richesse du kraal, c'est l'enfant. Celui qui a une importante progéniture, est hautement considéré et peut souvent exercer une grande influence sur les affaires publiques. L'enfant travaille très tôt et il est courant de voir un gamin de six ans, avec ses frères aînés, faire parcourir au bétail 20 kilomètres en un jour. A dix ans, on lui confie la garde des veaux, des moutons et des chèvres. Pendant ce temps, les filles apprennent leur métier de femme : transporter l'eau, ramasser le bois, traire etc.

Les parents ont tendance à retarder le mariage de leur fils: en effet, le jeune a un troupeau à lui, formé au long des ans depuis sa naissance, mais ces bêtes restent dans le troupeau paternel. Au moment du mariage d'un fils, le père a besoin de plusieurs têtes de bétail, comme biens matrimoniaux qui iront à la famille de la fille. Ce système de compensation fait que souvent un garçon ne se mariera que grâce aux bêtes reçues à l'occasion du mariage de sa soeur. L'intérêt des familles, c'est que les mariages soient bien équilibrés entre voisins et ennemis potentiels: ainsi les solidarités naturelles sont renforcées, les coups de main moins à craindre.

ères exercent un contrôle considérable sur les affaires de leurs enfants, même après qu'ils aient atteint l'âge adulte. Un pasteur doit le plus souvent attendre la mort de son père pour disposer comme il l'entend de tout ou partie du troupeau de la famille.

De leur côté, les fils désirent prendre leur indépendance, ils veulent se marier sans tarder, de sorte que leurs intérêts s'opposent souvent à celui des parents. Il n'est pas rare que les rapports entre père et fils soient tendus ; par la coutume, ils ne mangent pas ensemble. Ce qui n'empêche pas la confiance, une affection réciproque. Le père sait qu'un jour, il pourra compter sur l'aide de ses enfants et ceux-ci reconnaissent l'expérience pastorale du père.

48 Les jeunes sont donc mis au travail dès leur enfance, ils apprennent le métier en grandissant. Vivant ensemble au camp avec ceux de leur âge, ils gardent ensemble les bêtes en brousse, ce qui développe chez le Turkana le sens de la responsabilité et de la coopération.

49 Il n'est pas de vie sans eau! Nulle part dans le monde cette évidence n'est plus dramatique que chez les Turkana. Ils ne peuvent pas avoir de foyer fixe. Ils doivent suivre les nuages de pluie, aller d'un trou d'eau à un autre trou d'eau et, quand leurs dromadaires, leur bétail ont tondu les pâturages, se déplacer encore. Cette quête de pâturages et d'eau instaure un mode de vie particulier, épuisant pour hommes et bêtes.

50 La vie des jeunes gardiens suit donc le rythme des saisons: dispersion puis rassemblement. A la saison sèche, bergers et troupeaux s'en vont : les garçons sont libres, laissés à eux-mêmes. Par contre, la saison des pluies ramène tout le monde au camp de base : c'est alors l'époque des fêtes, des amours, des mariages, mais aussi des contraintes sociales. Le campement devient alors le théâtre de toutes les activités rituelles.

51 Contrairement à d'autres pasteurs-nomades, le campement de base ne suit pas tout le troupeau. La plus grande partie des bêtes, sous la direction des jeunes guerriers qui s'installent dans des campements mobiles, part pour plusieurs mois. Le reste du bétail demeure sur place et assure la subsistance des gens du campement de base ou awi napolon.

Civilisations, 41 | 2009 
Enfin, citons parmi les travaux féminins : traire, baratter, préparer la nourriture, laver la vaisselle de bois et de cuir, nettoyer le kraal, entretenir le feu, construire des abris. La femme Turkana a une vie moins éprouvante que sa soeur cultivatrice : elle est souvent mieux nourrie, avec un régime plus riche en protéines. On compare volontiers la femme à une vache. C'est que la femme, comme la vache, signifie permanence et croissance de vie.

\section{La passion pastorale} faut aimer le bétail pour supporter sa compagnie. Dans les conditions matérielles où se trouvent les Turkana, c'est une ascèse continuelle. En effet, la présence des bêtes attire les mouches et il faut se faire à l'odeur de la bouse. Pour éloigner les insectes, on entretient des feux ; mais la fumée finit par imprégner les gens et tout le matériel.

59 A l'image des garde-boeufs qui se posent parmi le bétail, le Turkana est souvent longiforme et patient : il peut se tenir debout pendant des heures, immobile, attentif à tout ce qui se passe dans les environs. Il a une âme de combattant : habitué à défendre son troupeau, il sait se servir d'une lance et son idéal est le guerrier qui n'a peur de rien. Endurant et sobre, il ignore le confort. Par fierté, il sait dominer ses besoins. Courageux, téméraire même, il n'en est pas moins un homme intelligent, rusé, expert en 
négociations. Il invente mille et une façon de berner l'autre. Il est maître dans l'art de parler sans jamais livrer sa pensée.

\section{Les rituels} en-dehors menaçant et en-dedans familier. La vie du Turkana se déroule dans un univers concret: la nature, connue certes, mais qui réserve toujours des surprises. C'est la brousse, avec ses montagnes, ses fauves, ses plantes ; c'est encore la voûte du ciel avec le soleil qui brûle, l'obscurité et ses pièges, la tornade qui arrose et l'éclair qui foudroie. La question primordiale est alors : comment la nature peut m'être favorable? ensemble. Une coutume illustre cette croyance: les Turkana enterrent leurs morts au milieu du kraal. La vie entière, du berceau à la tombe, se déroule sous les auspices du bovin. Le nouveau-né reçoit un nom bénéfique ; on évite de lui donner le nom d'un raté ; on choisit plutôt celui d'un parent qui a du prestige et on ajoute un surnom d'après les plus belles bêtes du troupeau. On éprouve la chance du nouveau-né en lui sacrifiant une chèvre. A l'occasion du mariage, on présente le troupeau du jeune homme. La famille de la jeune fille reçoit des têtes de bétail.

le l'akipeyore est le plus impressionnant. Tous les adultes initiés de la région se donnent rendez-vous et se réunissent sous un arbre sacré. L'aîné, marchant et brandissant sa lance, explique le pourquoi du sacrifice et finalement frappe l'animal de son arme. La façon dont l'animal tombe signifie l'acceptation ou le rejet de la victime et, dans ce dernier cas, il faudrait en immoler une autre. Après avoir mangé, l'hommemédecine (emuron) parle très longtemps, frappant le sol de son bâton pour donner de la force à ses phrases. Ce sacrifice est fait pour le bénéfice du groupe. Le sacrifice va être 
l'occasion de réunir les familles vivant dans l'adakar et de renforcer les liens socioéconomico-politiques.

\section{Les projets de 'développement' et l'avenir des Turkana}

Le Kenya moderne veut coûte que coûte alphabétiser les nomades du nord. Bonne initiative pourrait-on dire! Cependant, l'alphabétisation se fait par des 'étrangers' dans une langue étrangère, le Kiswahili. Les enfants Turkana n'ont aucun point de repère, car les cours ne sont pas adaptés à la réalité socio-économique. De plus, les instituteurs ne s'en cachent pas pour dénoncer le 'primitivisme' de leurs élèves. Nous connaissons plusieurs cas de jeunes qui refusent obstinément de poursuivre leur scolarisation parce qu'ils sont la risée de leurs camarades de classe. De plus, l'alphabétisation se fait pour une grande partie autour des missions et ceci a des conséquences sur plus d'un point. Comment alors alphabétiser convenablement ces jeunes pasteurs :

- à partir de la langue maternelle (mais le Kenya n'échappe pas non plus au syndrome du 'nation building' en utilisant une seule langue),

- un enseignement qui pourrait jeter un pont entre le mode de vie pastoral et le 'modernisme',

- une alternance école / vie au kraal.

Qui plus est, un enfant qui va à l'école, n'est pas remplacé par quelqu'un d'autre dans sa tâche économique, si petite soit-elle. La conséquence est la déstructuration de la société. Le gouvernement kenyan, avec l'aide de la coopération, a foré un peu partout dans la région turkana des puits modernes sans avoir fait une analyse anthropologique préalable. Les conséquences sont désastreuses :

- les membres des adakar se disputent les droits aux puits,

- vu la facilité relative d'obtenir de l'eau, les pasteurs restent plus longtemps confinés aux abords des puits avec un nombre surélevé de têtes de bétail. Le pays, déjà un désert, se désertifie à vue d'oeil,

- certaines régions ne sont plus utilisées par les pasteurs. La conséquence est la 'green desertification', c'est-à-dire que les plantes comestibles sont détruites par la végétation nuisible.

Il y a environ dix ans, un projet de pêche a vu le jour près de Kalakol. Ayant constaté que des milliers de Turkana s'adonnaient à la pêche artisanale (ils avaient perdu leurs troupeaux au début des années '60 suite à la grande sécheresse), le gouvernement a mis sur pied un projet de pêche industrielle. Le peu d'argent que certains Turkana avaient gagné, a été investi directement dans des têtes de bétail afin de réintégrer la société nomade. Ceux qui sont restés pêcheurs artisanaux sont devenus des parias de cette société. Le complexe de Kalakol, qui a coûté des millions, est en train de tomber en ruine par la rouille. Les hangars sont utilisés par les nomades en quête d'ombre aux heures les plus chaudes de la journée.

Les Turkana ne cultivent pas. Surtout au sud, le gouvernement essaie de sédentariser les pasteurs et de leur inculquer les principes de base d'une agriculture irriguée. La plupart des projets sont de véritables échecs.

71 Depuis quelques années, la région turkana est 'infestée' par des missions bibliques américaines et néerlandaises. Celles-ci essaient, avec n'importe quels moyens, d'évangéliser les Turkana qui sont considérés comme des sauvages sans foi ni loi. Des 
Turkana complètement appauvris et dépourvus de dignité 'nomadisent' maintenant autour des missions. Ces nouveaux prolétaires du nord-ouest kenyan sont à la merci des cultes dominicaux pour lesquels ils reçoivent une compensation en nourriture, surtout du maïs.

Que seront-ils demain? Les Turkana vivent en autarcie, maîtres d'eux-mêmes, tantôt riches, tantôt pauvres. Face aux calamités naturelles, ils manifestent une grande capacité d'adaptation. Ils se sont montrés peu perméables aux idées et aux pratiques du monde moderne. Non sans raison.

Pour les Turkana comme pour l'ensemble des peuples traditionnels, l'impact du monde moderne est terrible. Avec la colonisation puis les indépendances, de nouveaux pouvoirs sont apparus. Les états modernes ne tiennent pas à partager le pouvoir avec les pasteurs, à leur laisser leur autonomie; leur genre de vie même pose des problèmes: des populations sans demeure fixe échappent au contrôle de l'Etat, aux impôts et aux règlements. Les plans de 'développement' ne les atteignent que partiellement. Comment concilier la coutume pastorale d'abattre les bêtes seulement à l'occasion des sacrifices et le marché qui exige régulièrement une quantité de bétail pour la boucherie?

Menacés dans leur existence même, les Turkana ont refusé la nouveauté. Ils s'accrochent à leur genre de vie de toujours. Il faut avouer qu'ils ont réussi un équilibre difficile, une performance remarquable: élever du bétail aux confins du désert. Leur expérience séculaire leur permet de survivre, de bien vivre, là où la plupart des humains auraient renoncé.

\section{BIBLIOGRAPHIE}

BERBEN, p. \& WYMEERSCH, P., 1983 - De Turkana. Gebruiksvoorwerpen, kledij, sieraden en corporele decoratie bij de semi-sedentaire Turkana, Africa-Tervuren, XXIX, 1/2, p. 35-50.

BOGERS, K., WYMEERSCH p. \& BERBEN P., 1987 - In search of the Turkana monster. Ang'u or the reinforcement of social values. A hypothesis, Afrikanistische Arbeitspapiere, Schriftenreihe des Kölner Instituts für Afrikanistik, Universität zu Köln, 9, p. 51-76.

DEENEN, V., DIMMENDAAL, G., BOGERS, K., BERBEN, p. \& WYMEERSCH, P., 1988 - De Turkana. Herders van noord-west Kenia, Brochure uitgegeven ter gelegenheid van de Turkanatentoonstelling in het Rijksmuseum voor Volkenkunde te Breda (24 maart t/m 28 augustus), 32 p. WYMEERSCH, P., 1982 - Kledij en sieraden van de Turkana, Een kijk op, Informatietijdschrift van de Club Vlaamse Explorators-Ontdek de Wereld, Nr. 5, p. 1 \& 4.

WYMEERSCH, p. \& BERßEN, P., 1986 - The homestead of Nakoro. Some socio-cultural aspects of daily life among the Turkana of the Kerio region, Afrikanistische Arbeitspapiere, Schriftenreihe des Kölner Instituts für Afrikanistik, Universität zu Köln, 5, p. 97-120.

WYMEERSCH, p. \& BEKE, D., 1987 - The killing desert ? Droogte, nomadentradities en ontwikkelingsbestuur bij de Turkana, Afrika Focus, Tijdschrift van de AVRUG, Afrika Vereniging van de Rijksuniversiteit Gent, Nr. 3/4, p. 211-235. 
WYMEERSCH, P., 1988 - Turkana cattle classification. Some preliminary notes, Afrikanistische Arbeitspapiere, Schriftenreihe des Kölner Instituts für Afrikanistik, Universität zu Köln, 16, p. 123-148.

WYMEERSCH, P., 1991 - Ontwikkelingsprojecten en teloorgang van traditionele culturen : de Turkana van Kenia, Tribaal Nieuws, Afrika Special, WIP, Stichting Werkgroep Inheemse Volken, Amsterdam, Nr. 6, p. 5-9.

\section{RÉSUMÉS}

The Turkana, a pulation of around 250,000 nomadic shepherds, live in the north-west of Kenya in an extremely arid and barren area between lake Turkana and Uganda. Having lived many years without any intrusion of the modern world, the Turkana, nomadic shepherds perfectly adapted to their hostile environment, had to, however, diversify their pastoral economy following the serious drought of 1961 . Some of them took up fishing. The author, gives us a brief survey of the social and economic structures of this ethnic group. Concentrating on the place of cattle, both from a social and a symbolic point of view, within the Turkana community, he depicts the recent changes that have taken place in their way of life following the wish of the Kenyan government to 'develop' this region.

\section{AUTEUR}

\section{PATRICK WYMEERSCH}

Rijksuniversitair Centrum - College voor de Ontwikkelingslanden - Universiteit Antwerpen Antwerpen - Belgique 